\title{
Diacronie
}

Studi di Storia Contemporanea

$N^{\circ} 10,2$ | 2012

Digital History: la storia nell'era dell'accesso

\section{La lezione di Prometeo e il filologo nell'era digitale}

Rassegna di risorse informatiche per lo studio dell'antichità classica

\section{Gianluca Canè}

\section{(2) OpenEdition \\ Journals}

Edizione digitale

URL: http://journals.openedition.org/diacronie/2880

DOI: 10.4000/diacronie. 2880

ISSN: 2038-0925

Editore

Association culturelle Diacronie

Notizia bibliografica digitale

Gianluca Canè, "La lezione di Prometeo e il filologo nell'era digitale », Diacronie [Online], № 10, 2 | 2012, documento 9, Messo online il 29 juin 2012, consultato il 21 avril 2019. URL : http://

journals.openedition.org/diacronie/2880; DOI : 10.4000/diacronie.2880 


\title{
Diacronie
}

9/

\section{La lezione di Prometeo e il filologo nell'era digitale}

\section{Rassegna di risorse informatiche per lo studio dell'antichità classica*}

\author{
Gianluca CANÈ **
}

Il presente contributo intende offrire una breve panoramica delle risorse informatiche rivolte allo studio dell'antichità classica. In particolare, la rassegna prende in esame tre tipologie di strumenti: a) le applicazioni gratuite cross-platform che permettono di gestire al meglio la codifica dei caratteri Unicode e di comporre facilmente testi in greco politonico dalla tastiera del computer; b) i siti gratuiti di interesse generale per i classicisti, che danno accesso a lessici e dizionari, banche dati testuali e percorsi tematici su argomenti specifici; c) le collezioni di testi classici in formato digitale (Thesaurus Linguae Graecae, Thesaurus Linguae Latinae), che consentono di consultare un vastissimo corpus di opere greche e latine ed effettuare ricerche combinate sui testi. 


\section{Introduzione}

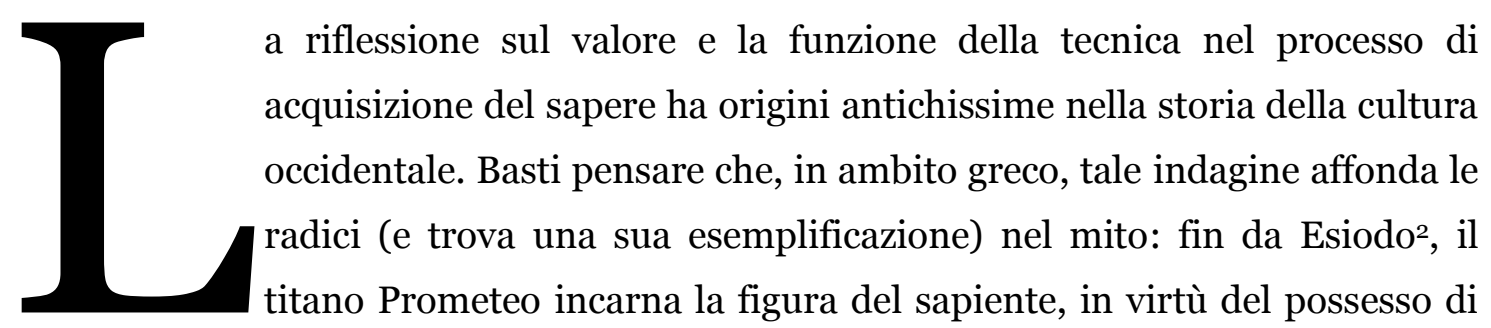

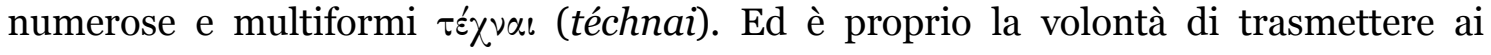
mortali la conoscenza di tali téchnai, con l'intento di offrir loro i doni riservati solamente agli dèi, a rendere il figlio di Giapeto - detentore di un sapere senza confini ${ }^{3}$ - oggetto della punizione divina, ma al contempo massimo benefattore dell'umanità.

L'episodio del furto del fuoco da parte di Prometeo, nel racconto mitologico, funge dunque da simbolo di un duplice progresso da parte del genere umano: l'affrancamento

Lettore, a lui solo va riconosciuto il merito. Di ogni imprecisione o errore di valutazione il sottoscritto è invece da ritenere l'unico responsabile.

${ }^{1}$ La citazione - che nella versione italiana della pellicola recita: «Mi sono trasformato d'incanto da classicista convinto a uno che si nutre di cultura popolare. Non credo che esista un'altra cultura. Insomma, la nostalgia non è cultura: quando si inizia a custodirle come reliquie, le usanze sono finite, obsolete» - è tratta dal film: LANG, Samantha, The monkey's mask, Fandango, Australia-Canada-Francia-Giappone-Italia, 2000, 93’.

${ }^{2}$ La vicenda di Prometeo è menzionata in due diversi contesti: ESIODO, Teogonia, vv. 507-616; Opere e giorni, vv. 42-105. Per tutte le traduzioni dei passi tratti dalle opere esiodee, citate di qui in avanti, ho utilizzato: ESIODO, Opere, testi introdotti, tradotti e commentati da Graziano Arrighetti, Torino, Einaudi-Gallimard, 1998.

3 Negli epiteti formulari dell'esametro esiodeo è rimarcata con grande frequenza la proverbiale

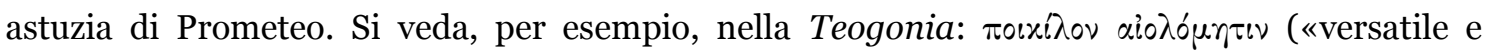

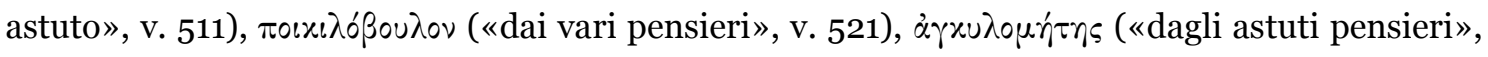

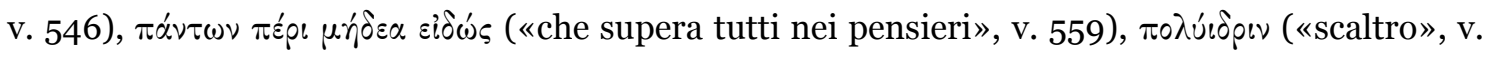

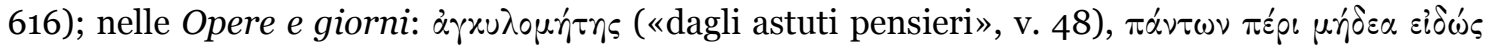
(«che supera tutti nei pensieri», v. 54).

Si ritrova in ESCHILO, Prometeo incatenato, v. 85, di cui si discuterà più approfonditamente in seguito, la testimonianza di una tradizione - risalente con ogni probabilità a fonti mitografiche più antiche - legata al "nome parlante" di Prometeo, ossia «il Preveggente» (da $\pi \rho 0-[p r o-]$, «in

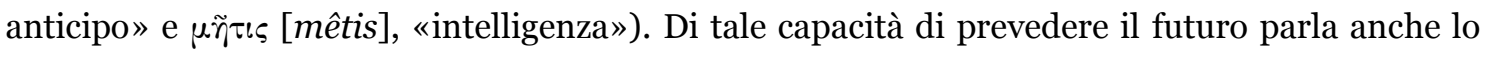

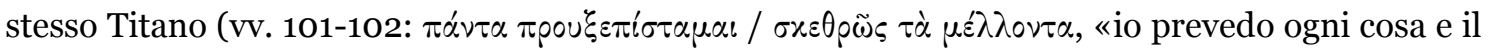
futuro è ben chiaro») che, in altri passi dell'opera, è definito $\delta \varepsilon เ v o ́ s$, ossia «formidabile» a trovare

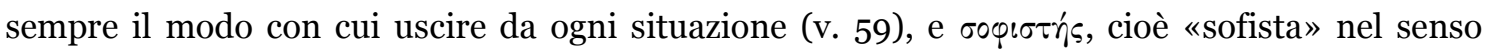
etimologico di «sapiente» (v. 62). 
dal divino e la conquista degli strumenti che consentono di prevalere sulla natura ${ }^{4}$. Il concetto è ben espresso nella narrazione tragica di Eschilo5, che - pur condividendo

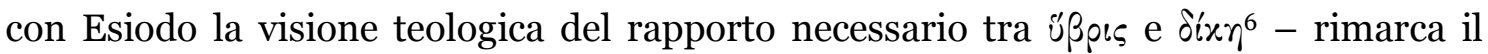
valore dell'azione salvifica di Prometeo per il miglioramento della condizione umana. L'apprendimento delle arti ${ }^{7}$ e della tecnica ha infatti permesso agli uomini di comprendere segreti che in precedenza consideravano imperscrutabili e appannaggio esclusivo della mente divina, oltre che di piegare (ancorché parzialmente) ${ }^{8}$ l'ambiente circostante ai propri scopi e alle proprie esigenze.

La conoscenza delle téchnai corrisponde in sostanza all'acquisizione di una coscienza e all'esercizio del pensiero9; e il dispiegamento della ragione, mediante la pratica del lógos, rappresenta il viatico verso l'emancipazione. Non è un caso, in tal

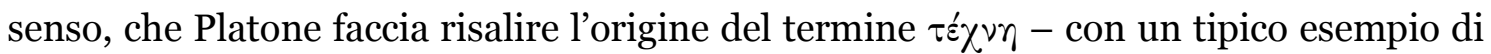

4 A proposito del mito di Prometeo e del suo forte valore simbolico, si veda GALIMBERTI, Umberto, Psiche e techne. L'uomo nell'età della tecnica, Milano, Feltrinelli, 1999, pp. 51-79.

5 ESCHILO, Prometeo incatenato, vv. 88-113; 193-255 (a proposito del furto del fuoco). Per tutte le traduzioni dei passi tratti dall'opera eschilea, citate di qui in avanti, ho utilizzato: ESCHILO, Le tragedie, traduzione, introduzioni e commento a cura di Monica Centanni, Milano, Mondadori, 2003.

${ }^{6}$ Faccio qui riferimento a quella norma tipica della teodicea arcaica, secondo la quale ad ogni atto di «superbia» nei confronti degli dèi (hýbris) corrisponde sistematicamente una giusta punizione atta a ristabilire la «giustizia» interna al cosmo (díke) e gli equilibri che regolano i rapporti tra divinità e genere umano. Basti citare in questa sede, a proposito dell'argomento, le celebri pagine di DODDS, Eric R., I Greci e l'irrazionale, Milano, Sansoni, 2003, pp. 71-84.

$7 \mathrm{Nel}$ dramma eschileo a Prometeo è attribuito il merito di aver insegnato ai mortali anche moltissime altre téchnai (ricordate ai vv. 436-506), tanto che il Titano ne conclude la rassegna

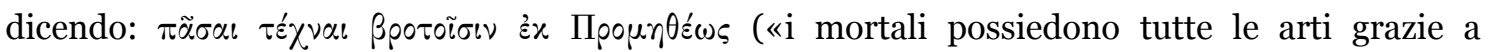
Prometeo», v. 506).

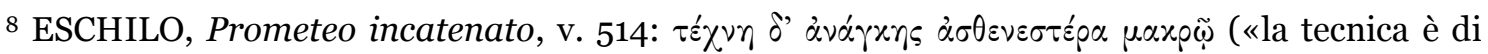
gran lunga meno potente della necessità»), dove per «necessità» - $\alpha \nu \alpha \dot{\gamma} \gamma \times \eta$ - si intende qui quel principio che «regola la natura e la scansione del suo ciclo che nessun progetto umano può infrangere e di fronte al quale ogni espediente tecnico dimostra il suo limite» (GALIMBERTI, Umberto, Psiche e techne. L'uomo nell'età della tecnica, Milano, Feltrinelli, 1999, pp. 51-52).

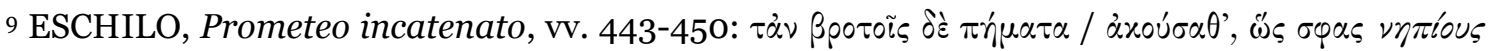

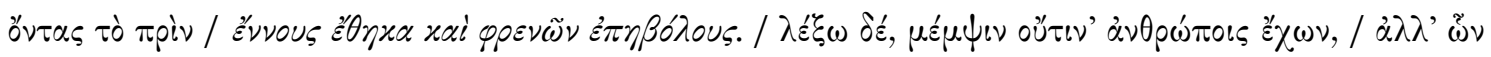

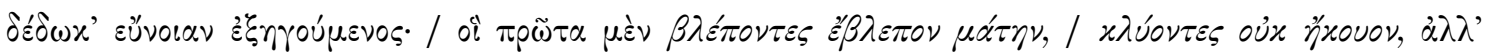

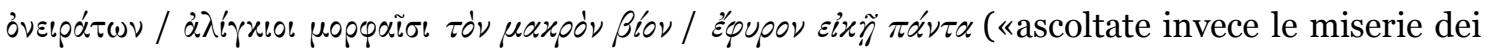
mortali: erano come infanti prima, e io diedi loro coscienza e pensiero. Parlerò senza disprezzo alcuno per gli uomini, ma vi spiegherò quanto fu il mio amore per loro, quali i miei doni. Prima, avevano occhi e non vedevano, orecchie e non sentivano, ma come le immagini nei sogni vivevano confusamente una vita lunga, inconsapevole»; miei i corsivi nel testo e nella traduzione). 


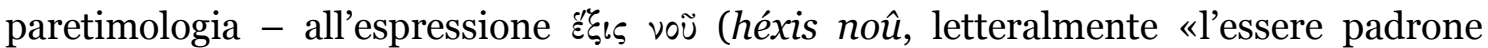
della propria mente») ${ }^{10}$ e che, per spiegare l'importanza della tecnica nella vita dell'uomo ${ }^{11}$, menzioni proprio il mito di Prometeo:

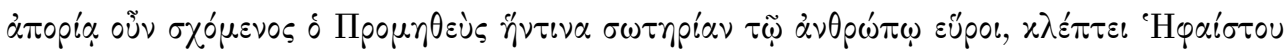

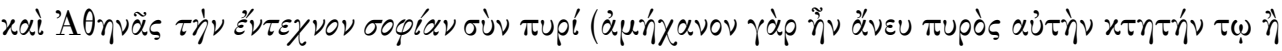

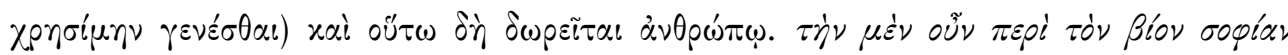

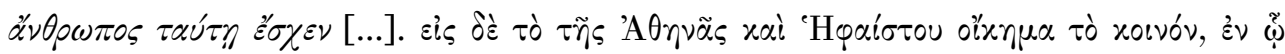

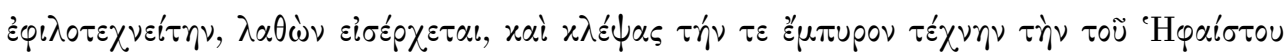

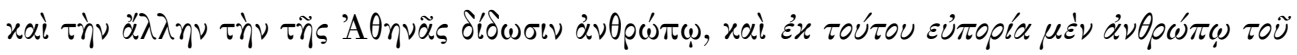

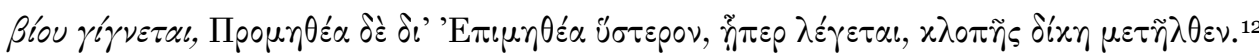

Ancora una volta, anche nel racconto platonico, la figura di Prometeo assurge a paradigma del progresso; un progresso, fondato sulla tecnica, tanto utile quanto necessario al miglioramento della condizione umana.

Con questa breve incursione nei testi greci antichi ho cercato di rintracciare - per così dire - un nobile precedente, allo scopo di dimostrare che anche chi lavora sui classici può ritrovare nei classici uno spunto di riflessione sulla necessità di integrare la scienza con la tecnica. Gli antichisti, per forma mentis, tendono talvolta ad essere refrattari all'utilizzo delle téchnai nello svolgimento delle proprie attività; il ricorso alle risorse informatiche in particolare viene guardato con sospetto dagli studiosi abituati, per formazione o pratica consolidata, a maneggiare con disinvoltura volumi ponderosi e a muoversi con agilità tra logori (quanto talvolta lacunosi) repertori cartacei. Ma ad oggi il filologo dell'età moderna non può più rinunciare all'immenso apporto che le tecnologie forniscono anche allo studio dell'antichità: prendendo a modello la lezione del Titano che conosceva tutte le téchnai e rimanendo sempre conscio dei limiti

10 PLATONE, Cratilo, 414b-c.

${ }^{11}$ Sul valore attribuito alle téchnai - considerate un modello del sapere - nei primi dialoghi platonici, si veda CAMBIANO, Giuseppe, Platone e le tecniche, Roma-Bari, Laterza, 1991, pp. 6184 e 85-115.

12 PLATONE, Protagora, 321c-322a («allora Prometeo, non sapendo quale mezzo di salvezza escogitare per l'uomo, ruba ad Efesto e ad Atena la loro sapienza tecnica, insieme al fuoco (senza il fuoco era infatti impossibile acquisire e utilizzare quella sapienza), e la dona all'uomo. In tal modo, l'uomo ebbe la sapienza tecnica necessaria per la vita [...]. Entra dunque furtivamente [scil. Prometeo] nell'officina di Atena ed Efesto, in cui essi praticavano insieme la loro tecnica, e, rubata la tecnica del fuoco di Efesto e quella di Atena, la dona all'uomo. Di qui vennero all'uomo le sue risorse per la vita. Ma Prometeo, a causa di Epimeteo, in seguito, come si narra, subì la pena per il furto»; miei i corsivi nel testo e nella traduzione). 
intrinseci alla tecnica, deve imparare a servirsi della tecnologia come strumento per semplificare e rendere più accurato il proprio lavoro; e trovato così un giusto connubio

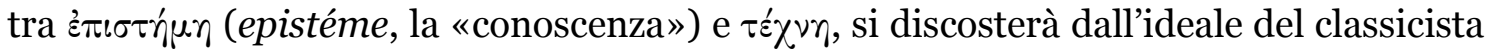
di stampo hermanniano ${ }^{13}$ per trasformarsi in un novello Prometeo. O, per meglio dire: in un umanista digitale.

Ma veniamo ora alla rassegna delle risorse di cui può disporre il classicista dei tempi moderni. La panoramica, lungi dall'essere esaustiva, è concepita per fungere soltanto da agile introduzione agli strumenti di base e si pone come unica «aspirazione, onesta quanto umile, di poter risultare in qualche modo utile» ${ }^{14}$ a chi voglia conoscere $\mathrm{i}$ rudimenti delle téchnai ancillari allo studio del mondo antico.

\section{Caratteri greci e videoscrittura}

Ogni studente che abbia avuto l'esigenza di dattiloscrivere lettere dell'alfabeto greco nel testo della propria tesi di laurea sa quanto la resa dei caratteri possa costituire un problema di difficile soluzione: oltre che in fase di inserimento, anche la modifica su computer diversi da quello personale o la stampa presso le tipografie presenta spesso notevoli difficoltà (per la mancanza del font dedicato o per la sua incompatibilità tra piattaforme differenti).

In passato costituiva un buon espediente - valido ancora oggi - l'impiego di una serie di azioni automatizzate (un set di “macro"), scritte in Visual Basic per Microsoft Office Word ${ }^{15}$, che permettevano di digitare i caratteri greci con relativi accenti e segni

${ }_{13}$ Mi riferisco al filologo tedesco Gottfried Hermann (1772-1848), riconosciuto primo fautore di un approccio sistematicamente formale alla filologia e capofila della cosiddetta scuola della Wortphilologie («filologia della parola»). Sull'attività di Hermann e sui termini della polemica con August Böckh (1785-1867), sostenitore di un metodo di studio di più ampio respiro della classicità nella sua complessità, si veda l'ottimo contributo di DEGANI, Enzo, «Filologia e storia», in Eikasmós. Quaderni Bolognesi di Filologia Classica, 10, 1999, pp. 279-314 (e in particolare le pp. 279-292).

${ }^{14}$ SANTORO, Marco, ORLANDI, Antonella, Avviamento alla bibliografia. Materiali di studio e di lavoro, Milano, Editrice Bibliografica, 2006, p. 253.

${ }^{15}$ Il sistema è stato elaborato dal Professor Francesco Citti dell'Università di Bologna ed è ancora oggi scaricabile dal sito del "Dipartimento di Filologia classica e Italianistica - FICLIT" dell'Ateneo bolognese. URL: < http://www.ficlit.unibo.it/biblioteca/collezionidigitali/strumenti-informatici-per-lantichita-classica/fonts-e-varie-utilita $>$ [consultato il 25 giugno 2012]. 
diacritici in maniera piuttosto intuitiva, in abbinamento a un font di simboli ${ }^{16}$. Ma $\mathrm{i}$ limiti imposti da questo pur ingegnoso sistema sono molteplici: la necessità di possedere una licenza di Microsoft Office Word aggiornata e di operare in ambiente VBA; la scarsa compatibilità fra diversi sistemi operativi del font, i cui glifi non corrispondono a nessuna lettera reale della mappa caratteri.

In realtà, $\mathrm{i}$ font di simboli sono una tecnologia superata da quando è nato e si è sviluppato lo standard Unicode. Unicode ${ }^{17}$ è un sistema di codifica, patrocinato dallo Unicode Consortium, che fornisce una mappatura universale e compatibile con qualunque piattaforma informatica dei 110.181 segni grafici propri delle principali lingue del mondo, fra cui anche il greco politonico esteso, oltre a numerosi simboli matematici; in altri termini, a ciascun glifo è associato un numero univoco che identifica in maniera specifica un dato carattere (per esempio, alpha con spirito dolce) indipendentemente dal font utilizzato e dal sistema operativo in uso ${ }^{18}$. In tal modo, è sempre garantita la corrispondenza tra il codice che definisce una lettera - non più un simbolo, come nel caso dei symbol font - e il suo equivalente grafico, oltre che la piena compatibilità cross-platform, soprattutto se si tiene conto che i testi scritti in Unicode possono essere decodificati da alcuni set di caratteri presenti in ogni sistema ("Arial Unicode MS”, “Palatino Linotype” e "Tahoma”), anche in assenza del font utilizzato originariamente per digitare il documento.

Ovviamente esistono font Unicode elaborati specificamente per la miglior resa grafica possibile dell'alfabeto greco esteso e, a questo proposito, la scelta è determinata soltanto dal gusto estetico personale. Fra i siti che forniscono gratuitamente una vasta raccolta di font da scaricare segnalo:

- le Alan Wood's Unicode resources, disponibili all'indirizzo < http://www.alanwood.net/unicode/ $>$ 19, che riportano la mappatura integrale dei caratteri specifici di ogni lingua compresa nello standard Unicode, la lista dei font reperibili in Rete (gratuitamente o a pagamento) suddivisi per lingua e sistema operativo di preferenza, una ricca sezione di applicazioni e strumenti per la digitazione dei caratteri speciali da tastiera, una serie di utili indicazioni sugli

\footnotetext{
${ }^{16}$ Si tratta del font Greek. Per "font di simboli" (symbol font) si intende un set di caratteri i cui glifi non sono interpretati dal computer come vere e proprie lettere, ma come semplici pittogrammi.

17 URL: < http://www.unicode.org/ > [consultato il 25 giugno 2012].

18 Per una descrizione del funzionamento di Unicode e dello sviluppo dei suoi standard, si consulti l'URL: < http://www.unicode.org/consortium/newcomer.html > [consultato il 25 giugno 2012].

19 Consultato il 25 giugno 2012.
} 
standard da seguire per pubblicare testi contenenti caratteri Unicode in ambiente web e un'ampia sitografia;

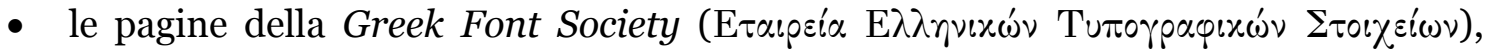
raggiungibili all'indirizzo < http://www.greekfontsociety.gr/index.html $>^{20}$, che nella sezione "Typefaces" del sito raccolgono un buon numero di font professionali disegnati per la scrittura dell'alfabeto greco - visualizzabili in anteprima e suddivisi per epoca e stile -, con i link diretti per il download dei file;

- le Greek pages di Russell Cottrell, disponibili all'indirizzo < http://www.russellcottrell.com/greek/unicode.htm $>^{21}$, in cui è possibile reperire una breve storia della nascita e sviluppo dello standard Unicode, insieme a una raccolta significativa di caratteri visualizzabili in anteprima (con link diretti ai siti dei rispettivi produttori per il download dei file) e una utile sitografia;

- la sezione dedicata ai font greci della WAZU JAPAN's Gallery of Unicode Fonts, disponibile all'indirizzo < http://www.wazu.jp/gallery/Fonts_GreekPoly.html >22.

In abbinamento a un set di caratteri specifico per la resa grafica delle lettere greche, è consigliabile dotarsi (per una gestione fluida della codifica Unicode) di un softwaretastiera, ossia di uno strumento che consenta di passare facilmente da un alfabeto all'altro e di digitare i singoli caratteri - soprattutto se accentati o comprensivi di segni sovrasegmentali - in maniera intuitiva.

In quest'ambito, si distingue per praticità un'applicazione compatibile con i sistemi operativi Windows di nome "Keyman Desktop", sviluppata dalla Tavultesoft ${ }^{23}$ e acquistabile ad una modica cifra sul sito del produttore ${ }^{24}$. I criteri di scrittura sono molto semplici da memorizzare: dopo aver inserito il carattere corrispondente a una vocale, basta digitare - singolarmente o in combinazione - alcuni simboli facilmente rintracciabili sulla tastiera e il sistema aggiunge automaticamente spiriti e/o accenti sulla lettera ${ }^{25}$, convertendola nel glifo equivalente secondo la mappatura Unicode.

\footnotetext{
${ }^{20}$ Consultato il 25 giugno 2012.

${ }^{21}$ Consultato il 25 giugno 2012.

${ }^{22}$ Consultato il 25 giugno 2012.

23 URL: < http://www.tavultesoft.com/ > [consultato il 25 giugno 2012].
}

24 In passato esisteva una versione del software distribuita gratuitamente per utilizzo personale, oggi purtroppo non più disponibile. Per ottenere comunque una versione free della risorsa, si veda, URL: < http://help.keymandesktop.com/docs/common_free.php > [consultato il 25 giugno 2012].

${ }^{25}$ Le parentesi quadre producono gli spiriti, dolce con ']' e aspro con '['; gli slash aggiungono invece gli accenti, acuto con '/' e grave con 'l', mentre il segno '=' corrisponde al circonflesso. 
Merita invece una menzione particolare l'unica risorsa gratuita e multipiattaforma atta a questo scopo, ovvero "Thessalonica"26. Si tratta di un'utilità implementata nel linguaggio Java dal bizantinista Alexej Kryukov, distribuita sotto forma di pacchetto di estensione .uno per Apache OpenOffice ${ }^{27}$ e LibreOffice ${ }^{28}$; il sistema comprende anche un utile strumento di conversione che permette di modificare la codifica dei caratteri 8 bit allo standard Unicode o viceversa. Per poter utilizzare a pieno le funzionalità di "Thessalonica 3.1", è necessario dotarsi di OpenOffice.org 2.1 (o versioni successive: si consiglia l'ultima release stabile, che al momento della stesura di questo articolo è Apache OpenOffice 3.4.0) o LibreOffice e del Java Runtime Environment 1.6 (JRE) ${ }^{29}$.

Una volta scaricato e installato il file dell'estensione, “Thessalonica 3.1" si presenta sotto forma di barra degli strumenti nel menu di Writer. L'interfaccia grafica consta di quattro parti: lo strumento di personalizzazione del programma, l'icona che monitora lo stato della tastiera, il menu che consente di selezionare la lingua da utilizzare per la scrittura e infine il pulsante di controllo del convertitore di codifica per i caratteri. Occorre a questo punto configurare una tantum le impostazioni preferite che si desidera adottare ogni qual volta venga utilizzata l'applicazione. Selezionare quindi il primo elemento ("Customize keyboard") e, nella nuova finestra a comparsa, fare attenzione ai seguenti campi:

- Layout Parameters. Definisce il metodo di inserimento dei caratteri (o layout di tastiera) che deve essere utilizzato dal sistema per comporre i testi nelle diverse lingue; nel caso del greco politonico Unicode, la voce da selezionare è necessariamente "Greek Ibycus".

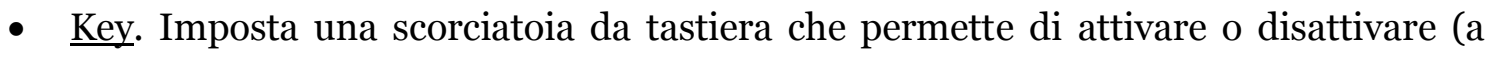
seconda del layout in uso in quel momento) con la stessa combinazione di tasti la

Digitando '+' è possibile inserire la dieresi al di sopra delle vocali iota e upsilon, mentre ' ' fa comparire lo iota sottoscritto. Per esempio, una sequenza come 'a[/l' (dove '[//' possono essere digitati in qualsiasi ordine) darà origine a \%ُ, cioè alpha con spirito aspro, accento acuto e iota sottoscritto.

${ }^{26}$ URL: < http://www.thessalonica.org.ru/en/index.html > [consultato il 25 giugno 2012].

27 Per informazioni sul progetto Apache OpenOffice, recentemente donato dalla Oracle alla Apache Software Foundation, si veda il sito ufficiale, URL: < http://www.openoffice.org/it/ > [consultato il 25 giugno 2012].

${ }_{28}$ Per informazioni sulla nascita di The Document Foundation e sullo sviluppo della suite LibreOffice, nata da una costola del progetto OpenOffice.org dopo l'acquisizione da parte della Oracle, si veda il sito ufficiale, URL: < http://it.libreoffice.org/ > [consultato il 25 giugno 2012]. 29 Per verificare la presenza e il corretto funzionamento della piattaforma Java sul proprio computer ed eventualmente installare una copia del software, si veda il sito ufficiale, URL: < http://www.java.com/it/download/ > [consultato il 25 giugno 2012]. 
funzione di inserimento dei caratteri greci o occidentali e di passare così da un alfabeto all'altro; in questo caso è possibile assegnare la combinazione ritenuta più comoda e intuitiva in rapporto alle proprie esigenze e preferenze.

- Font Parameters. Associa un set di caratteri specifico per gli alfabeti speciali utilizzati dalle lingue in cui si desidera comporre i testi, definendone anche lo stile ("Bold", grassetto, o "Italic", corsivo) e la dimensione espressa in punti ("Size”); anche in questo caso è possibile scegliere il font Unicode e le formattazioni preferite, tenendo conto della dimensione e degli stili assegnati di default al testo normale per non creare squilibri.

- Language. Attiva la funzione del controllo ortografico della lingua speciale digitata attraverso il metodo di inserimento caratteri prescelto; nel caso del greco politonico, si consiglia di disattivare questa opzione - scegliendo la voce "No language" dal menu a cascata - dal momento che Writer non prevede un dizionario di greco antico in grado di verificare la correttezza ortografica e grammaticale del testo inserito.

Per quanto riguarda i criteri di digitazione dei segni diacritici, il metodo di inserimento è molto simile a quello previsto dal software "Keyman Desktop" descritto sopra: le parentesi tonde producono gli spiriti, dolce con ')' e aspro con '('; l'apostrofo aggiunge l'accento acuto, il segno "' 30 l'accento grave e '=' corrisponde al circonflesso; infine, digitando '+' è possibile inserire la dieresi al di sopra delle vocali iota e upsilon, mentre 'l' fa comparire lo iota sottoscritto e '!' il sottopunto per identificare le lettere incerte (nei papiri o nelle iscrizioni).

Complessivamente, "Thessalonica 3.1" si presenta come lo strumento più duttile per comporre agilmente testi in greco politonico dalla tastiera del computer, in virtù delle sue molte caratteristiche positive: come componente aggiuntivo gratuito di Apache OpenOffice o LibreOffice, si integra con un'applicazione di videoscrittura freeware, open-source e compatibile con qualunque sistema operativo (Windows, Mac OS o Linux); è di facile installazione e configurazione; i metodi di inserimento dei segni speciali sono molto intuitivi e facili da memorizzare ${ }^{31}$.

30 Il simbolo " " si ottiene: in ambiente Windows, con la combinazione Alt+96 (da tastierino numerico); in ambiente Mac OS, con Alt $+\backslash$; in ambiente Linux, con AltGr+' (apostrofo).

${ }^{31} \mathrm{~A}$ margine di queste considerazioni, segnalo agli utenti più esperti anche la possibilità di personalizzare i criteri di inserimento dei caratteri, mediante la modifica dei file di configurazione di "Thessalonica 3.1" (il codice sorgente dell'applicazione è disponibile nella sezione "Download" del sito dello sviluppatore, segnalato supra, nota 26). Per ulteriori 


\section{I classici online: strumenti lessicali, testi e percorsi tematici}

Nel mare magnum della Rete, non sempre è semplice districarsi tra la pletora di informazioni cui è possibile attingere, anche perché spesso i requisiti fondamentali che una risorsa deve possedere per essere ritenuta valida dallo storico - l'attendibilità e l'affidabilità - non sono rispettati.

Partendo dall'aspetto linguistico, non è superfluo ricordare che il web mette a disposizione utili repertori lessicografici. I grecisti e, in generale, gli studiosi di letteratura interessati alla storiografia o alla storia della lingua greca possono accedere gratuitamente alla versione elettronica integrale della $S u d a$, l'imponente enciclopedia del mondo antico elaborata in ambiente bizantino intorno al X secolo. Il progetto Suda On Line (SOL) ${ }^{32}$, nato nel 1998 e in continuo sviluppo, si avvale della collaborazione di centinaia di esperti grecisti e informatici in tutto il mondo e si pone l'obiettivo di «compilare una banca dati codificata nel linguaggio XML, dotata di strumenti di ricerca testuale e navigabile in Rete, che comprenda un corredo di annotazioni costantemente arricchite, di riferimenti bibliografici e collegamenti ipertestuali ad altre risorse elettroniche, in aggiunta alla traduzione letterale delle singole voci della Suda»33.

Ad oggi, la traduzione inglese dei 30.000 lemmi del lessico è quasi completata, ma le note e i commenti sono aggiornati quotidianamente. Fra le caratteristiche aggiuntive del portale, si segnala la possibilità di:

- effettuare ricerche sull'intera banca dati utilizzando numerosi campi (per lemma, per traducente, per riferimento numerico all'edizione originale Adler, per parola chiave, ecc.);

- visualizzare il testo greco in diverse codifiche, compresa quella Unicode;

- seguire i rimandi bibliografici e i link ai materiali di corredo interni ottenendo una panoramica di tutte le occorrenze in cui la risorsa selezionata è citata;

- controllare il significato di una parola greca sulla versione online del dizionario Liddell-Scott-Jones (LSJ) ${ }^{34}, \quad$ disponibile all'indirizzo,

URL: <

istruzioni e indicazioni in tal senso, si rimanda direttamente alla ricchissima user guide che accompagna l'applicazione.

${ }^{32}$ URL: < http://www.stoa.org/sol/ > [consultato il 25 giugno 2012].

33 Mia la traduzione dall'inglese di questo estratto dalla pagina di informazioni sul progetto, consultabile sul sito ufficiale all'indirizzo, URL: < http://www.stoa.org/sol/about.shtml > [consultato il 25 giugno 2012].

34 LIDDELL, Henry George, SCOTT, Robert, A Greek-English Lexicon, revised and augmented throughout by Sir Henry Stuart Jones, with the assistance of Roderick McKenzie, Oxford, Clarendon Press, 1940. 
http://www.perseus.tufts.edu/hopper/text?doc=Perseus\%3atext\%3a1999.04.0057 $>35$.

Quest'ultimo riferimento ci consente di citare anche altri dizionari e lessici di lingue classiche - consultabili liberamente in Rete - disponibili sul portale Perseus, di cui si parlerà più approfonditamente in seguito a proposito delle banche dati a testo pieno. Per la lingua greca si segnala:

- la versione ridotta del Liddell-Scott-Jones (LSJ) ${ }^{36}$, disponibile all'indirizzo, URL: < http://www.perseus.tufts.edu/hopper/text?doc=Perseus\%3Atext\%3A1999.04.0058 $>37$;

- l'edizione elettronica, disponibile per la consultazione all'indirizzo, URL: < http://www.perseus.tufts.edu/hopper/text?doc=Perseus\%3Atext\%3A1999.04.0073 $>38$, del lessico omerico di base curato dallo Autenrieth ${ }^{39}$;

- l'edizione elettronica, disponibile per la consultazione all'indirizzo, URL: < http://www.perseus.tufts.edu/hopper/text?doc=Perseus\%3Atext\%3A1999.04.0072 $>4^{40}$, del lessico pindarico curato dallo Slater ${ }^{41}$.

Per il latino si segnala:

- l'edizione elettronica, disponibile per la consultazione all'indirizzo, URL: < http://www.perseus.tufts.edu/hopper/text?doc=Perseus\%3Atext\%3A1999.04.0059 $>{ }^{42}$, del dizionario curato da Lewis e Short (LLD) ${ }^{43}$;

- la versione ridotta del Lewis-Short (LLD)44, disponibile all'indirizzo, URL: < http://www.perseus.tufts.edu/hopper/text?doc=Perseus\%3Atext\%3A1999.04.006 $\mathrm{O}>45$.

35 Consultato il 25 giugno 2012.

${ }^{36}$ LIDDELL, Henry George, SCOTT, Robert, An Intermediate Greek-English Lexicon, Oxford, Clarendon Press, 1889.

37 Consultato il 25 giugno 2012.

${ }^{38}$ Consultato il 25 giugno 2012.

39 AUTENRIETH, Georg, A Homeric Dictionary for Schools and Colleges, New York, Harper and Brothers, 1891.

${ }^{40}$ Consultato il 25 giugno 2012.

${ }^{41}$ SLATER, William J., Lexicon to Pindar, Berlin, De Gruyter, 1969.

${ }^{42}$ Consultato il 25 giugno 2012.

43 LEWIS, Charlton T., SHORT, Charles (revised, enlarged, and in great part rewritten by), $A$ Latin Dictionary (LLD), founded on Andrews' edition of Freund's Latin dictionary, Oxford, Clarendon Press, 1879. 
Tutti gli strumenti qui menzionati - passando ora a discutere delle banche dati testuali - si integrano con una risorsa imprescindibile per i classicisti, che riassume in sé i principi fondamentali del cosiddetto "umanesimo digitale" (accessibilità, gratuità, compatibilità, interoperabilità e multimedialità): il portale Perseus.

Descrivere in poche parole le caratteristiche della Perseus Digital Library ${ }^{46}$, sviluppata dalla Tufts University del Massachusetts (USA) fin dal 1985, è impresa ardua: si tratta in sostanza di un'opera enciclopedica, che abbraccia la storia, la letteratura e - in senso lato - la cultura dei popoli del bacino del Mediterraneo (Greci, Romani e Arabi). Il progetto è focalizzato soprattutto sul mondo greco-latino e si pone l'obiettivo di raccogliere il corpus integrale delle testimonianze di civiltà (fonti letterarie, reperti archeologici, contesti storici) prodotte in quell'area47. Ma il sito offre moltissimi materiali anche per lo studio dei popoli germanici, sulla storia del Nord America nel XIX secolo, sulla letteratura inglese moderna e sulla poesia di matrice umanistico-rinascimentale prodotta in Italia fra il XIII e il XVI secolo48.

La collezione di testi greci e latini disponibile su Perseus, codificata interamente in Unicode, è davvero imponente, come si può notare sfogliando la sezione "Greek and Roman Materials", dove ogni opera è accompagnata da una traduzione integrale inglese tratta da edizioni non recenti (sempre segnalata in una nota bibliografica a piè pagina). Ma ciò che rende eccezionale questa banca dati è la possibilità di eseguire ricerche a tutto campo - sui testi o sugli apparati di corredo - e di selezionare ogni singolo termine che compare in un testo per ottenere una immediata analisi grammaticale, cercarne il significato sui dizionari di lingue classiche presenti sul sito e rintracciare

44 LEWIS, Charlton T., An Elementary Latin Dictionary, New York-Cincinnati-Chicago, American Book Company, 1890.

45 Consultato il 25 giugno 2012.

${ }^{46}$ URL: < http://www.perseus.tufts.edu/hopper/ > [consultato il 25 giugno 2012].

47 Per ulteriori ragguagli sulla mission e i contenuti del progetto, si veda la pagina di informazioni sul sito ufficiale, consultabile all'indirizzo, URL: < http://www.perseus.tufts.edu/hopper/research > [consultato il 25 giugno 2012].

48 Imprescindibile, per quanto riguarda la letteratura umanistica e i testi in latino d'età rinascimentale in prosa o in poesia, è anche il Philological Museum dell'Università di Birmingham (Regno Unito), consultabile liberamente online all'indirizzo, URL: < http://www.philological.bham.ac.uk/ > [consultato il 25 giugno 2012]. Comprende invece anche le fonti più antiche l'ottimo Archivio della Latinità Italiana del Medioevo (ALIM), un progetto frutto di una partnership fra l'Università Statale di Milano, la Ca' Foscari di Venezia, la Federico II di Napoli, e gli Atenei di Verona, Siena-Arezzo e Palermo - che si propone di raccogliere e rendere accessibili l'insieme dei testi latini prodotti in Italia nel corso del Medioevo: si veda, URL: < http://www.uan.it/alim/ > [consultato il 25 giugno 2012]. 
tutte le occorrenze di quella voce (indipendentemente da come sia declinata o coniugata) all'interno della stessa opera o di tutta la biblioteca digitale ${ }^{49}$.

Non meno ricca, anche se non propriamente incentrata sul concetto di interattività, è l'utilissima biblioteca di testi greci e latini digitalizzati raccolta da Ulrich Harsch, la Bibliotheca Augustana ${ }^{50}$. Il sito, in realtà, raccoglie opere che risalgono a contesti culturali molto diversi tra loro: si ritrovano infatti sezioni dedicate alla letteratura tedesca, anglosassone, francese, italiana, spagnola, portoghese, polacca, ceca, russa ed ebraica. Il punto di forza di questo repertorio consiste nell'ottima suddivisione dei materiali, cui è sempre possibile accedere attraverso un indice alfabetico o cronologico, nella cura con la quale vengono citate le fonti cartacee originali utilizzate per acquisire i testi, nella attenta selezione di riferimenti bibliografici o link di interesse menzionati a proposito di ogni autore e singola opera proposta, e nel rigido rispetto degli standard Unicode per la codifica dei caratteri greci, un accorgimento che consente di copiare ed esportare facilmente in qualunque programma di videoscrittura ampie porzioni di testo.

Altre raccolte (per la verità, non molto ordinate) di opere greche e latine, con o senza traduzione, possono essere reperite fra le sezioni di un'ultima tipologia di strumenti, che citeremo qui - per concludere la panoramica - a proposito delle risorse di carattere generale. Si tratta di siti che fungono da collettori di materiali aventi per tema la classicità in senso lato, su cui è possibile trovare riferimenti bibliografici o approfondimenti tematici suddivisi per autore, periodo cronologico o genere letterario. Tra i portali più ricchi di informazioni, è opportuno menzionare:

\footnotetext{
49 La possibilità di ottenere una lista delle concordanze, partendo da una parola contenuta in un testo, è fornita anche dalla IntraText Digital Library, che offre un'ampia sezione dedicata alla latinità (dall'antichità al XXI secolo): URL, < http://www.intratext.com/LATINA/ >. Una pecca di questo repertorio consiste nel fatto che spesso non è citata l'edizione cartacea originale da cui è stato prodotto il testo elettronico e questo aspetto rende difficile stabilirne la reale affidabilità. Questo stesso difetto si ritrova anche in altre tre rassegne di testi latini, che citerò per completezza, ma che necessitano di essere utilizzare con grande scrupolo: la Bibliotheca Latina di Domenico Parro (URL: < http://www.romaeterna.org/fabulae/bib-lt.html > [consultato il 25 giugno 2012]), in cui è fornito - laddove possibile - anche il link a una traduzione disponibile in Rete dell'opera selezionata; The Latin Library (URL: < http://www.thelatinlibrary.com/ > [consultato il 25 giugno 2012]); il Corpus Scriptorum Latinorum raccolto da David Camden (URL: < http://www.forumromanum.org/literature/index.html > [consultato il 25 giugno 2012]).

50 URL: < http://www.hs-augsburg.de/ harsch/augustana.html > [consultato il 25 giugno 2012].
} 
- Pomoerium ${ }^{51}$, in lingua inglese, che raccoglie tutte le ultime novità in ambito di studi classici, dalle bibliografie aggiornate sugli autori e sulle opere della letteratura greco-latina ai più recenti studi critici di papirologia, epigrafia, storiografia e linguistica. Non manca poi un'area riservata ai testi e una utile sezione dedicata ai link di interesse per i classicisti (riviste specialistiche, archivi e motori di ricerca);

- L'antiquité grecque et latine ${ }^{52}$, in lingua francese, che abbraccia tutti gli aspetti più importanti della letteratura classica. Fornisce traduzioni integrali di testi greci e latini, manuali di storia letteraria, dizionari mitologici, griglie riassuntive degli autori e delle opere, tavole esplicative, percorsi tematici e tantissimi collegamenti a risorse utili per lo studio del mondo antico.

- la Rassegna degli Strumenti Informatici per lo Studio dell'Antichità Classica53, curata da Alessandro Cristofori, che costituisce un ottimo punto di partenza per esplorare ciò che offre la Rete sulla storia greca e romana. Nel corso di dodici anni di lavoro - il progetto è stato avviato nel 1995 e aggiornato fino al 2007 - il curatore ha raccolto una quantità enorme di risorse di tipo informatico: non solo siti web, ma anche CD-ROM e software. Le fonti trattate sono quelle letterarie, epigrafiche, papiracee, numismatiche, relative a documentazione e a immagini archeologiche o cartografiche, a cui si aggiungono riviste e biblioteche elettroniche e siti dedicati a mostre e musei, anche virtuali. I servizi catalogati, disponibili in formato digitale, sono repertori bibliografici su disco e in linea, informazioni su strutture scientifiche presenti in Rete, nonché su indirizzi, convegni e posizioni lavorative, gruppi di discussione, software specifici. Altre sezioni, infine, sono dedicate ai materiali didattici consultabili direttamente online.

\section{Banche dati a testo pieno e programmi di interrogazione}

Non potremmo concludere questa rassegna senza citare una tipologia di strumenti di indiscussa rilevanza nell'ambito degli studi classici: le banche dati testuali disponibili su CD-ROM. Tra le più importanti e di più largo utilizzo, ricordiamo:

\footnotetext{
${ }^{11}$ URL: < http://www.pomoerium.eu/index.html > [consultato il 25 giugno 2012].

${ }^{2}$ URL: < http://remacle.org/ > [consultato il 25 giugno 2012].

53 URL: < http://www.rassegna.unibo.it/ > [consultato il 25 giugno 2012]. Purtroppo dal 2007 il sito non è più aggiornato, ma rappresenta ancora oggi un catalogo utilissimo per reperire informazioni circostanziate e ben curate su tutti gli aspetti della classicità.
} 
- il Thesaurus Linguae Graecae (TLG)54, una vera e propria biblioteca digitale della letteratura greca implementata a partire dal 1972 dalla Università di Irvine in California, che contiene testi letterari da Omero al XV secolo d.C.;

- il Phackard Humanities Institute (PHI)55 \#5, equivalente latino del TLG prodotto a Los Altos in California, che raccoglie testi letterari dalle origini fino al 200 d.C., con una selezione di autori posteriori e alcuni testi biblici56;

- $\quad$ il Phackard Humanities Institute (PHI) \#7, la versione su supporto magnetico della Duke Data Bank of Documentary Papyri (DDBDP)57, che comprende un vasto corpus di documenti scritti su papiri, óstraka e tavolette lignee, oltre a un'ampia selezione di testi epigrafici d'età classica e cristiana in lingua greca e latina.

Tutti e tre questi repertori necessitano di essere abbinati ad un programma di interrogazione esterno, che consenta di ricercare all'interno del database e di ottenere i risultati in base ai parametri inseriti. A questo scopo, esistono molti software, venduti separatamente, compilati per ogni sistema operativo ("Musaios"58 e "Lector"59 per Windows; "Pandora"6o e "SNS-Greek \& Latin"61 per Mac OS).

54 URL: < http://www.tlg.uci.edu/ > [consultato il 25 giugno 2012]. L'ultima versione pubblicata su CD-ROM, nell'anno 2000, è contrassegnata dalla lettera E, ma dal 2004 ogni ulteriore edizione aggiornata è distribuita ed è consultabile soltanto online, dietro sottoscrizione di una licenza; per una cronistoria completa del progetto TLG, si veda la pagina di informazioni all'indirizzo, URL: < http://www.tlg.uci.edu/about/history.php > [consultato il 25 giugno 2012]. 55 URL: < http://www.packhum.org/info.html > [consultato il 25 giugno 2012].

${ }^{56}$ La copertura della produzione letteraria tardoantica, originariamente prevista per una nuova edizione della banca dati, è ora stata abbandonata. Un utile elenco delle opere incluse nel CDROM PHI 5, comprendente anche le edizioni utilizzate per la digitalizzazione dei testi, si trova sul sito Internet dell'“Electronic Text Resource Service” dell'Università dell'Indiana all'indirizzo, URL: < http://www.indiana.edu/ letrs/text-tools/textlists/phibibliog.html > [consultato il 25 giugno 2012].

${ }^{57} \mathrm{Si}$ tratta di una banca dati testuale sviluppata dall'Università di Duke a Durham (USA). Per consultare i documenti online si veda, URL: < http://papyri.info/browse/ddbdp/ > [consultato il 25 giugno 2012]. Per informazioni sulla storia e il contenuto del progetto, si veda, URL: < http://idp.atlantides.org/trac/idp/wiki/DDBDP > [consultato il 25 giugno 2012].

${ }^{58}$ Sviluppato da Darl Dumont e Randall Smith (Los Angeles, California). Per informazioni, si veda, URL: < http://www.musaios.com/ > [consultato il 25 giugno 2012].

59 Sviluppato da Robert Maier (Freising, Germania). Per informazioni, si veda, URL: < http://www.maierphil.de/lector/index.html > [consultato il 25 giugno 2012].

60 Il software è mantenuto e distribuito dalla American Philological Association (Università della Pennsylvania, Philadelphia), a cui è possibile inoltrare ordini di acquisto via email. Per informazioni, si veda, URL: < http://www.apaclassics.org/ > [consultato il 25 giugno 2012]. 
Tuttavia, l'applicazione più funzionale e diffusa per la piattaforma Windows - che qui illustreremo brevemente - è senza dubbio il pacchetto "Workplace" commercializzato dalla Silver Mountain Software (Texas, USA) ${ }^{62}$. Utilizzando questo software, è infatti possibile:

- leggere a video uno dei testi del TLG, PHI \#5 o PHI \#7, selezionando un autore e/o un'opera dalla lista alfabetica;

- spostarsi all'interno dell'opera prescelta mediante il cursore o inserendo gli estremi di un passo preciso dal menu a pulsante "Ref";

- evidenziare qualsiasi parola di un testo e aprire la relativa scheda lessicografica sulla versione informatizzata dei dizionari delle lingue classiche (Liddell-ScottJones per il greco e Lewis-Short per il latino) oppure vederne l'analisi morfologica grazie al collegamento con le pagine Internet del sito di Perseus ${ }^{63}$;

- selezionare, copiare e incollare porzioni di testo ${ }^{64}$ in un qualsiasi programma di videoscrittura;

- effettuare ricerche sull'indice delle forme attestate all'interno dell'intera banca dati, specificando se una particolare stringa deve trovarsi all'inizio, alla fine di una parola o esservi semplicemente contenuta ${ }^{65}$;

${ }^{61}$ Sviluppato dal "Laboratorio di Scienze dell'Antichità" (LILA) della Scuola Normale Superiore di Pisa. Per informazioni, si veda, URL: < http://snsgreek.sns.it/sns_02.html > [consultato il 25 giugno 2012].

${ }^{62}$ Per informazioni, si veda, URL: < http://www.silvermountainsoftware.com/workpack.html > [consultato il 25 giugno 2012].

${ }^{63}$ Sulla Perseus Digital Library in generale e sugli strumenti di analisi lessicale e morfologica in essa integrati, cfr. supra, § 2.

64 Si tenga conto che la banca dati del TLG, PHI \#5 e PHI \#7 è codificata originariamente in un linguaggio chiamato BetaCode, che utilizza i primi 128 caratteri ASCII. Tale formato, di per sé poco pratico, può tuttavia essere interpretato e convertito automaticamente dal software "Workplace" nella codifica Unicode: in questo modo, oltre ai vantaggi sulla resa grafica del testo da visualizzare a video, sarà possibile esportare brani leggibili con qualunque applicazione e compatibili con tutti i sistemi operativi. Per configurare "Workplace" in maniera corretta, aprire il menu "Setup" dalla finestra principale del programma, fare clic su "Preferences" e accedere alla scheda "Language Display": spuntare a questo punto la casella che si trova in corrispondenza della voce "Use Unicode Greek", salvare le impostazioni e riavviare l'applicazione.

${ }^{65}$ In questo, come in tutti i successivi casi citati, i risultati della ricerca vengono ordinati da "Workplace" in un elenco di occorrenze opera per opera: ogni voce della prima lista ottenuta è a sua volta cliccabile per vedere ulteriori dettagli e da ogni singolo item selezionato è possibile aprire l'opera a testo pieno, per analizzare ciascuna occorrenza nel suo contesto più ampio. L'indice dei risultati (comprensivi dei riferimenti ad autore e opera, insieme a qualche riga di testo) è poi esportabile in un file .rtf a parte. 
- ricercare nessi o gruppi di parole nell'intera banca dati, in un gruppo di autori, in un singolo autore o in un'opera specifica, servendosi degli operatori logici AND $\left({ }^{*}\right)$, AND THEN (il semplice spazio bianco), OR (|) o NOT (!);

- creare indici e concordanze (anche inversi o frequenziali) di singoli autori o di singole opere, con l'opportunità di salvare il documento ottenuto in formato .rtf;

- per quanto riguarda il TLG, interrogare la banca dati impostando come criteri di ricerca i filtri del "canone" (genere letterario, periodo cronologico, area geografica) oppure creando liste personalizzate di autori.

Mi preme ricordare, in conclusione, che le stesse funzionalità - e anche qualcuna aggiuntiva, soprattutto per quel che riguarda la gestione della codifica del testo in vista dell'esportazione - sono previste da un altro software completamente gratuito e distribuito in pacchetti compatibili con qualunque piattaforma (Windows, Mac OS e

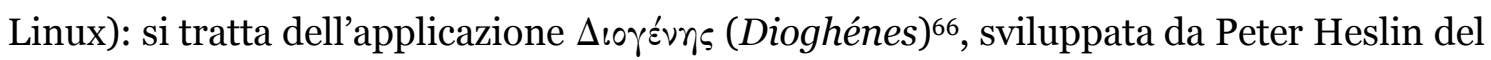
“Dipartimento di Studi Classici e Storia Antica” dell'Università di Durham (Regno Unito). Il programma, scritto in Perl, possiede una grande flessibilità che consente a chi abbia una certa pratica del linguaggio di personalizzare le modalità di ricerca e la formattazione dei risultati, ed è dotato di un'interfaccia web che permette di utilizzare "Diogenes" collegandosi ad un server. Nella piena osservanza dello spirito dell'opensource, gli utenti sono incoraggiati a modificare, migliorare e distribuire l'applicazione, nel rispetto delle normative in tema.

Tra le caratteristiche più rimarchevoli del programma ${ }^{67}$, illustrate nella pagina principale del progetto, si ricorda la connessione al motore di analisi morfologica di Perseus per tutti i vocaboli in greco e latino, la possibilità di effettuare ricerche attraverso l'indice del TLG e l'opportunità di scelta tra numerosi formati di esportazione dei testi greci (tra gli altri BetaCode, Ibycus per LaTeX, SMK GreekKeys, SIL Composite, SPIonic, Sgreek, SuperGreek per Mac OS, Unicode per la visualizzazione nelle pagine HTML, WinGreek, immagini .gif).

Termina qui questo excursus sugli strumenti informatici concepiti per affiancarsi alle risorse tradizionali nello studio dell'antichità classica. Inutile ricordare che le

${ }^{66}$ URL: < http://www.dur.ac.uk/p.j.heslin/Software/Diogenes/index.php > [consultato il 25 giugno 2012].

${ }^{67}$ La lista completa delle caratteristiche di "Diogenes" è consultabile all'indirizzo, URL: < http://www.dur.ac.uk/p.j.heslin/Software/Diogenes/features.php > [consultato il 25 giugno 2012]. 
téchnai - in quanto tali - devono essere utilizzate cum grano salis e sempre con la dovuta cautela: nessuna tecnologia potrà o dovrà mai sostituirsi alla preparazione, alla sensibilità e all'intuizione di uno studioso. Ma il sentiero verso l'acquisizione delle tecniche è ormai tracciato: impugni la lanterna, come Diogene ${ }^{68}$, anche il filologo classico e vada in cerca non già dell'uomo, ma delle nuove vie che conducono verso l'umanesimo digitale.

${ }^{68}$ Il celebre aneddoto su Diogene Cinico, che con una torcia accesa in pieno giorno si aggirava per le vie di Megara alla ricerca dell'uomo, è riportato da DIOGENE LAERZIO, Vite dei filosofi, VI 41. 


\section{** L'autore}

Gianluca Canè è dottore magistrale in Lettere Classiche. Si è occupato soprattutto di letteratura greca e in particolare di problemi della tradizione testuale dei testi antichi, tecniche di traduzione, letteratura frammentaria (scoliografica e lessicografica) e storia della storiografia antica, conseguendo la laurea in Storiografia greca presso l'Università di Bologna. Dal 2008 si interessa alle applicazioni delle risorse informatiche nello studio delle discipline classiche, collaborando con il Dipartimento di Filologia Classica e Medioevale a un progetto di digitalizzazione di un manuale di lingua greca e allo sviluppo di una banca dati sulla permanenza del classico. Dal 2009 è consulente della Zanichelli Editore SpA e dal 2011 è webmaster di Diacronie.

URL: < http://www.studistorici.com/2012/01/o4/gianluca-cane/ >

\section{Per citare questo articolo:}

CANĖ, Gianluca, «La lezione di Prometeo e il filologo nell'era digitale. Rassegna di risorse informatiche per lo studio dell'antichità classica», Diacronie. Studi di Storia Contemporanea: Digital History: la storia nell'era dell'accesso, 29/6/2012,

URL:<http://www.studistorici.com/2012/06/29/cane_numero_10/ >

\section{Diacronie Studi di Storia Contemporanea $\beta$ www.diacronie.it}

Risorsa digitale indipendente a carattere storiografico. Uscita trimestrale. redazione.diacronie@hotmail.it

Comitato di redazione: Marco Abram - Giampaolo Amodei - Jacopo Bassi - Luca Bufarale - Alessandro Cattunar - Alice De Rensis Barbara Galimberti - Deborah Paci - Fausto Pietrancosta - Matteo Tomasoni - Luca Zuccolo

Diritti: gli articoli di Diacronie. Studi di Storia Contemporanea sono pubblicati sotto licenza Creative Commons 2.5. Possono essere riprodotti a patto di non modificarne i contenuti e di non usarli per fini commerciali. La citazione di estratti è comunque sempre autorizzata, nei limiti previsti dalla legge. 